\title{
UTILIZAÇÃO DE PRÓTESE PARCIAL REMOVÍVEL OVERLAY NA REABILITAÇÃO ORAL: REVISÃO NARRATIVA
}

\author{
USE OF REMOVABLE PARTIAL PROSTHETIC OVERLAY IN ORAL \\ REHABILITATION: NARRATIVA LITERATURE REVIEW
}

\section{Jefferson David Melo de Matos', Ana Larisse Carneiro Pereira ${ }^{2}$, Ítalo Kennedy Silva Santos', Jeanne Maria Melo de Matos ${ }^{3}$, John Eversong Lucena de Vasconcelos ${ }^{4}$, Viviane Maria Gonçalves de Figueiredo ${ }^{5}$}

Autora para correspondência: Viviane Maria Gonçalves de Figueiredo - vivi_mfigueiredo@yahoo.com.br
'Graduando em Odontologia no Centro Universitário Doutor Leão Sampaio (UNILEÃO). Juazeiro do Norte, Ceará, Brasil.
${ }^{2}$ Graduanda em Odontologia no Centro Universitário Doutor Leão Sampaio (UNILEÃO). Juazeiro do Norte, Ceará, Brasil.
${ }^{3}$ Bacharel em Odontologia pelo Centro Universitário Doutor Leão Sampaio (UNILEÃO). Juazeiro do Norte, Ceará, Brasil.
Professor no Centro Caririense de Pós-Graduação (CECAP). Juazeiro do Norte, Ceará, Brasil.
${ }^{4}$ Mestre em Implantodontia. Doutorado em andamento em Implantodontia no Centro de Pesquisas Odontológicas São Leopoldo Mandic (SLMANDIC).
Poutora em Odontologia Restauradora. Professora no Centro Universitário Doutor Leão Sampaio (UNILEÃO). Juazeiro do Norte, Ceará, Brasil.

RESUMO I A busca incansável da Odontologia em reabilitar pacientes, proporcionou uma melhoria na qualidade de vida da população. Esse estudo objetiva demonstrar a importância do uso das Próteses Parciais Removíveis Provisórias do tipo Overlay na reabilitação oral de pacientes, por meio de uma revisão de literatura. Utilizou-se das principais bases de dados eletrônicas de catalogação bibliográfica (Pubmed, Scielo, BVS), no período de 2000 a 2016, com os seguintes descritores de assunto: Dimensão vertical, Prótese Parcial Removível, Revestimento de Dentadura. Foram localizados 35 artigos, dos quais 16 foram excluídos por não se enquadrarem nos critérios estabelecidos para inclusão, resultando no total de dezenove 19 artigos selecionados. As próteses parciais removíveis overlay são opções de tratamento reversível, as alterações nas estruturas dentais remanescentes são mínimas, clinicamente é fácil de ser executada, além do custo e tempo serem menores. Embora existam benefícios, cuidados devem ser tomados quanto à higienização oral e da prótese, aplicações tópicas de flúor e consultas de retorno ao consultório. Conclui-se que as PPR-overlay são utilizadas para pacientes com desgaste dentário acentuado associado à alteração da dimensão vertical de oclusão. Em contrapartida, estudos ainda precisam ser realizados a fim de assegurar a efetividade e viabilidade desse tipo de prótese, uma vez que, não são aplicáveis em todos os casos clínicos.

Palavras-chave: Dimensão vertical. Prótese Parcial Removível. Revestimento de Dentadura.

\begin{abstract}
The relentless pursuit of Dentistry in rehabilitating patients, provided an improvement in the quality of life of the population. This study aims to demonstrate the importance of the use of Removable Temporary Partial Dentures to of the Overlay type in the oral rehabilitation of patients through a review of the literature. It was used the main electronic databases of cataloging bibliography (Pubmed, Scielo, BVS), in the period 2000 to 2016 , employing the following descriptors: Vertical Dimension, Removable Partial Prosthetic, Coating of Dentures. Were identified 35 articles, 16 of which were excluded because they did not fit the established criteria for inclusion, resulting in a total of nineteen 19 selected articles. The removable partial prosthetic overlay is areversible treatment option, the changes in structures of the tooth remnants are minimal, clinically it is easy to be performed, in addition to the reduced cost and time to be smaller. Although there are benefits, care should be taken with the oral hygiene of the prosthesis, topical applications of fluoride and frequent visits to the dentist. It is concluded that the PPR-overlay are used for patients with accentuated dental wear associated with the change of the vertical dimension of the occlusion, on the other hand, studies still need to be carried out in order to ensure the effectiveness and feasibility of this type of prosthesis, once, they are not applicable in all clinical cases.
\end{abstract}

Keywords: Vertical Dimension. Removable Partial Denture. Overlay denture. 


\section{INTRODUÇÃO}

A busca incansável da Odontologia por reabilitar pacientes inválidos orais, proporcionou uma melhoria na qualidade de vida da população. Diversos são os aspectos a serem levados em consideração, são eles: a escolha do paciente, a seleção do profissional, a condição de higiene oral, limite biológico, qualidade de vida, aptidão do profissional e os custos a serem gastos com o tratamento'.

A confecção da PPR provisória, previamente à sua instalação, deve ser realizado primeiramente o preparo de boca 1 (um), esse consiste no conjunto de procedimentos na busca de um meio bucal adequado para receber a prótese, seguido do preparo de boca 2 (dois), ou seja, a instalação da PPR propriamente dita ${ }^{2}$.

As próteses parciais removíveis (PPR) são aparelhos destinados a substituir um ou mais dentes ausentes, devolvendo estética, fonética e função mastigatória. Podem ser removidas da boca pelo paciente ou profissional, exibem uma estrutura metálica que permitem a permanência da prótese em posição, conferem um mínimo preparo dos dentes e são de fácil higienização, em contrapartida a estética é comprometida ${ }^{3}$.

São utilizadas em extremidades livres uni ou bilaterais, espaços protéticos extensos e múltiplos, presença de perda óssea excessiva, necessidade de substituição imediata dos dentes, pacientes com fissuras palatinas (comunicação bucosinusal), odontopediatria e período de osseointregração dos implantes ${ }^{4}$.

A área protética considerada ideal consiste nas regiões livres de condições patológicas. Devem ser observadas outras variáveis, tais como a relação entre os arcos, dimensão e configuração do rebordo alveolar apropriadas, ausência de irregularidades no rebordo, quantidade de mucosa queratinizada adequada e profundidade de fundo de vestíbulo ${ }^{5}$.

Esse estudo tem como objetivo, demonstrar a importância do uso das Próteses Parciais Removíveis Provisórias do tipo Overlay na reabilitação oral de pacientes, por meio de uma revisão narrativa de literatura.

\section{MATERIAL E MÉTODOS}

Esse estudo utilizou as principais bases de dados eletrônicas de catalogação bibliográfica como: PUBMED (www.pubmed.gov), SCIELO (www.scielo. org), BVS (www.bvsalud.org), no período de 2000 a 2016, com os seguintes descritores de assunto: Dimensão vertical, Prótese Parcial Removível, Revestimento de Dentadura.

Foram catalogados trinta e cinco (35) artigos, dos quais dezesseis (16) foram excluídos por não se enquadrarem nos critérios de inclusão estabelecidos para a inserção dos artigos. Apenas trabalhos de revisão narrativa e/ou revisão sistemática da literatura e relatos de casos clínicos foram incluídos. Desta forma, a busca bibliográfica resultou em dezenove (19) artigos selecionados.

\section{REVISÃO DISCUTIDA DA LITERATURA}

Imensuráveis são as medidas realizadas para se obter um correto posicionamento do complexo maxilo mandibular que é o objetivo principal da reabilitação oral, pois as perdas dos elementos dentários resultam em uma desarmonia do sistema estomatognático, podendo resultar em modificações no padrão de oclusão normal, mudanças quanto à estética, fonética, harmonia facial e durante $o$ ato da mastigação ${ }^{\circ}$.

A dimensão vertical corresponde ao espaço intermaxilar em diferentes situações de abertura bucal, podendo se apresentar de duas formas. A primeira, como a dimensão vertical de repouso (DVR), definida como o espaço intermaxilar obtido no equilíbrio de contração mínima dos músculos elevadores e abaixadores da mandíbula. Ela coincide com a distância entre a comissura labial e determina um espaço entre os dentes. A segunda, como a dimensão vertical de oclusão (DVO), relação da mandíbula com a maxila em que os dentes encontra-se em contato, estabelecido pela contração dos músculos elevadores da mandíbula ${ }^{7}$.

Nos pacientes que exibem uma DVO reduzida é possível observar diminuição do terço inferior 
da face, protrusão do mento, intrusão dos lábios, severa profundidade dos lábios nasogenianos, perda de uma estabilidade da oclusão na região posterior, desgastes dentários acentuados, por uma perda da estrutura dental devido o contato excêntrico dos antagonistas (Atrição), musculatura hipotônica, fusão do lábio superior, ainda é visto na literatura comprometimentos na articulação Temporomandibular (ATM) e dos músculos envolvidos no processo mastigatório ${ }^{8}$.

Quando a distância equivalente entre a maxila e a mandíbula se encontra reduzidas, a tendência são os lábios se estenderem mais, formando uma prega na região de comissura labial, isso promove um acúmulo de saliva e proporciona um ambiente úmido, favorecendo assim a proliferação microbiana, e por sua vez o desenvolvimento de queilites angulares?

As PPR overlay podem ser utilizadas em qualquer tratamento, seja ele temporário ou definitivo, são indicadas para pacientes que tem uma dificuldade de se reestabelecera DVO e obter um alinhamento correto do plano oclusal. Esse dispositivo é definido como um instrumento protético removível que recobre o terço oclusal dos dentes posteriores e quando na região anterior reveste o terço incisal, permitindo um maior apoio e restaurando a oclusão funcional de todos os elementos da arcada. Auxilia no diagnóstico, prognóstico e durante o planejamento e execução do tratamento de reabilitação, permitindo assim uma avaliação tanto do padrão estético como funcional ${ }^{10}$.

Em casos mais complexos no quais se faz necessário o tratamento integrado, junto com outras especialidades, quando a prótese não é suficiente para sanar os problemas que afligem o paciente, um tratamento reabilitador se torna mais invasivo, complexo, a longo prazo e com custos relativamente mais altos que o convencional ${ }^{11}$.

Por isso é que para os pacientes que exibem uma condição financeira limitada e que tem uma baixa exigência estética, o tratamento com a PPR Overlay é a melhor alternativa reabilitadora. Portanto, - uso da PPR overlay é indicada para pacientes que apresentam DVO reduzida, podendo estar ou não associadas com restaurações, de forma que esses aparelhos permitem a recuperação da DVO, recuperando o plano oclusão original e abstraindo parte da sintomatologia dolorosa que estava interligada com a oclusão do paciente ${ }^{12}$.

\begin{tabular}{|c|c|c|c|}
\hline Autor/ano & Periódico publicado & Tipo de estudo & Síntese \\
\hline $\begin{array}{c}\text { Batista AUD ef al., } \\
2011^{1}\end{array}$ & $\begin{array}{c}\text { Pesquisa Brasileira em } \\
\text { Odontopediatria e Clínica } \\
\text { Integrada }\end{array}$ & Estudo Transversal & $\begin{array}{c}\text { De } 120 \text { modelos, } 92(76,7 \%) \\
\text { não apresentaram } \\
\text { planejamento, } 86 \text { modelos } \\
(71,7 \%) \text { foram classificados } \\
\text { como Pobre, } 23 \text { modelos } \\
(19,2 \%) \text { como boa e apenas } \\
11 \text { modelos }(9,2 \%) \text { como } \\
\text { Aceitável. }\end{array}$ \\
\hline $\begin{array}{c}\text { Goyatá FR et al., } \\
2008^{2}\end{array}$ & $\begin{array}{c}\text { International Journal of } \\
\text { Dentistry }\end{array}$ & Relato de Caso Clínico & $\begin{array}{l}\text { A evolução dos sistemas } \\
\text { restauradores com } \\
\text { propriedades mecânicas e } \\
\text { estéticas cada vez melhores, } \\
\text { tem-se a possibilidade de } \\
\text { novas alternativas para uma } \\
\text { reabilitação estética e } \\
\text { funcional. }\end{array}$ \\
\hline $\begin{array}{c}\text { Dekon SFC et al., } \\
2003^{4}\end{array}$ & $\begin{array}{c}\text { Revista Odontológica de } \\
\text { Araçatuba }\end{array}$ & Relato de Caso Clínico & $\begin{array}{l}\text { Paciente com bruxismo, } \\
\text { desgaste dental generalizado, } \\
\text { perda de guia anterior e } \\
\text { dimensão vertical de oclusão } \\
\text { apresentou bons resultados } \\
\text { quando reabilitado com } \\
\text { overlay e após } 40 \text { dias } \\
\text { condiçốes favoráveis para a } \\
\text { reabilitação com fixa. }\end{array}$ \\
\hline
\end{tabular}




\begin{tabular}{|c|c|c|c|}
\hline Autor/ano & Periódico publicado & Tipo de estudo & Síntese \\
\hline $\begin{array}{l}\text { Souza JEA ef al., } \\
2009^{5}\end{array}$ & $\begin{array}{c}\text { A Revista Odontológica do } \\
\text { Brasil-Central }\end{array}$ & $\begin{array}{l}\text { Revisão Integrativa e } \\
\text { Relato de Caso Clínico }\end{array}$ & $\begin{array}{l}\text { PPR overlay pode ser indicada } \\
\text { como alternativa de } \\
\text { tratamento para situaçóes } \\
\text { especiais de arcadas } \\
\text { parcialmente desdentadas, em } \\
\text { pacientes com necessidade de } \\
\text { alteração da dimensão } \\
\text { vertical e/ou realinhamento do } \\
\text { plano oclusal, podendo ser } \\
\text { empregada como tratamento } \\
\text { temporário ou definitivo. }\end{array}$ \\
\hline $\begin{array}{l}\text { Fragoso WS et al., } \\
2005^{\circ}\end{array}$ & $\begin{array}{l}\text { Revista Gaúcha de } \\
\text { Odontología }\end{array}$ & Relato de Caso Clínico & $\begin{array}{l}\text { Após os procedimentos de } \\
\text { acrilização e de instalação da } \\
\text { prótese, foi possivel concluir } \\
\text { que a prótese parcial } \\
\text { removível overlay apresentou } \\
\text { adequado suporte da } \\
\text { dimenção vertical de oclusão, } \\
\text { proporcionando conforto } \\
\text { funcional e satisfação ao } \\
\text { paciente. }\end{array}$ \\
\hline Neto $A F$ et al., $2011^{7}$ & $\begin{array}{l}\text { Odontologia Clínico- } \\
\text { Científica }\end{array}$ & $\begin{array}{c}\text { Revisão Sistemática da } \\
\text { Literatura }\end{array}$ & $\begin{array}{l}\text { A redução do edentulismo } \\
\text { observada nas últimas } \\
\text { décadas, mostra que a } \\
\text { demanda por PPR continuará } \\
\text { presente devido ao aumento } \\
\text { na expectativa de vida da } \\
\text { população e ao modelo } \\
\text { assistencial existente, o qual } \\
\text { ainda está longe de extinguir } \\
\text { a perda dentária. }\end{array}$ \\
\hline $\begin{array}{c}\text { Farias } A B L \text { et al., } \\
2009^{8}\end{array}$ & Brazilian Dental Science & Estudo de Coorte & $\begin{array}{l}\text { Apesar da prevalência de } \\
\text { DTM nos pacientes com } \\
\text { alteração da DVO, estas } \\
\text { variáveis não apresentaram } \\
\text { associação, não evidenciando } \\
\text { relação entre alteração da } \\
\text { DVO e presença de DTM. }\end{array}$ \\
\hline Rodrigues RA, $2010^{\circ}$ & $\begin{array}{c}\text { International Journal of } \\
\text { Dentistry }\end{array}$ & Relato de Caso Clínico & $\begin{array}{l}\text { A recuperação da altura do } \\
\text { terço inferior da face foi } \\
\text { conseguida com o emprego de } \\
\text { próteses de recobrimento } \\
\text { oclusal do tipo overlay } \\
\text { utilizadas pela paciente por } \\
\text { um período de tempo até que } \\
\text { cumprisse o período de } \\
\text { adaptação à nova DV. }\end{array}$ \\
\hline Cura $C$ et al., $2002^{10}$ & Quintessence Intertional & Relato de Caso Clínico & $\begin{array}{l}\text { Quando a dimensão vertical } \\
\text { for significativa, próteses } \\
\text { provisórias deve ser usado } \\
\text { antes da prótese final, para } \\
\text { prevenir o stress ou dor nas } \\
\text { articulaçóes } \\
\text { temporomandibulares e nos } \\
\text { músculos permitindo que o } \\
\text { paciente se adapte às novas } \\
\text { dimensões. }\end{array}$ \\
\hline
\end{tabular}




\begin{tabular}{|c|c|c|c|}
\hline Autor/ano & Periódico publicado & Tipo de estudo & Síntese \\
\hline $\begin{array}{l}\text { Patel MB et al., } \\
\qquad 2009{ }^{n}\end{array}$ & $\begin{array}{c}\text { The Open Dentistry } \\
\text { Journal }\end{array}$ & Relato de Caso Clínico & $\begin{array}{l}\text { A PPR overlay pode fornecer } \\
\text { um tratamento reversivel e } \\
\text { relativamente barato para } \\
\text { pacientes com um status dental } \\
\text { comprometida, embora não } \\
\text { exista muita evidência } \\
\text { científica na literatura sobre } \\
\text { sua confiabilidade. }\end{array}$ \\
\hline $\begin{array}{l}\text { Freitas } R \text { ef al., } \\
\qquad 2006^{12}\end{array}$ & $\begin{array}{l}\text { Revista Gaúcha de } \\
\text { Odontologia }\end{array}$ & Relato de Caso Clínico & $\begin{array}{l}\text { A PPR tipo overlay pode ser } \\
\text { considerada uma técnica } \\
\text { reabilitadora adequada em } \\
\text { situaçốes de redução de } \\
\text { colapso da dimensão vertical. }\end{array}$ \\
\hline $\begin{array}{l}\text { Feltrin PP ef al., } \\
\qquad 2008^{13}\end{array}$ & $\begin{array}{l}\text { Revista de Odontologia da } \\
\text { Universidade de São Paulo }\end{array}$ & Revisão de Literatura & $\begin{array}{l}\text { Mesmo com o avanço dos } \\
\text { métodos e das técnicas } \\
\text { Empregadas no registro da } \\
\text { dimensão vertical, ainda } \\
\text { nenhum dos métodos existentes } \\
\text { é cientificamente exato. As } \\
\text { determinações das dimensões } \\
\text { verticais, pelos clínicos, } \\
\text { parecem se basear em } \\
\text { experiências pessoais. }\end{array}$ \\
\hline $\begin{array}{l}\text { Pavarina } A C \text { ef al., } \\
2001^{14}\end{array}$ & $\begin{array}{l}\text { Journal of Prosthetic } \\
\text { Dentistry }\end{array}$ & Relato de Caso Clínico & $\begin{array}{l}\text { Próteses parciais removíveis } \\
\text { interino fabricadas para } \\
\text { estabelecer uma dimensão } \\
\text { vertical de oclusão terapêutica } \\
\text { aceitável, seguido de próteses } \\
\text { parciais removíveis de } \\
\text { sobreposição definitivo e } \\
\text { restaurações de resina } \\
\text { composta. }\end{array}$ \\
\hline $\begin{array}{l}\text { Kliemann } \mathrm{C} \text { ef al., } \\
2000^{15}\end{array}$ & $\begin{array}{l}\text { Revista Brasileira de } \\
\text { Prótese Clínica e } \\
\text { Laboratorial }\end{array}$ & Relato de Caso Clínico & $\begin{array}{l}\text { A Prótese Parcial Removível } \\
\text { Overlay pode ser indicada } \\
\text { como alternativa de } \\
\text { tratamento para situaçóes } \\
\text { especiais de arcadas } \\
\text { parcialmente desdentadas. }\end{array}$ \\
\hline $\begin{array}{l}\text { Marco RAC et al., } \\
2001^{16}\end{array}$ & $\begin{array}{l}\text { Revista Brasileira de } \\
\text { Prótese Clínica e } \\
\text { Laboratorial }\end{array}$ & Relato de Caso Clínico & $\begin{array}{c}\text { Tratamento reversivel, além de } \\
\text { apresentar menor custo e } \\
\text { menor tempo operacional. }\end{array}$ \\
\hline
\end{tabular}

Quadro 1. Descrição dos resultados encontrados nos trabalhos utilizados no estudo em questão.

Quando o assunto é reabilitação oral em pacientes parcialmente desdentados diversos são os tratamentos disponíveis, porém a efetividade e viabilidade das próteses parciais removíveis ainda precisam de mais estudos, uma vez que, não são aplicáveis a todos os $\operatorname{casos}^{13}$.

As próteses parciais overlays, de um modo geral, podem ser classificadas quanto à estrutura, material utilizado no revestimento oclusal e quanto à função.
Estudos mostram que o uso de superfícies metálicas podem aumentar o impacto da oclusão, prejudicando os dentes antagonistas, ligamento periodontal e osso de suporte, ainda que o uso de dentes de porcelana é consequência de resultados insatisfatórios com - uso da resina acrílica. Quanto à função, pode ser temporária, definitiva e terapêutica, a última quando utilizada apenas no período necessário para remissão de sintomas $^{14}$. 
Portanto, as overlays são indicadas para pacientes que apresentem desgaste dentário acentuado associado à alteração da dimensão vertical de oclusão, com o objetivo de se restabelecer o plano oclusal, na restauração do equilíbrio oclusal e como auxilio para diagnóstico e prognóstico, resultando na estabilização oclusal e condicionamento muscular ${ }^{15}$.

Os benefícios do uso das overlays são diversas, uma vez que, são procedimentos reversíveis, as alterações nas estruturas dentais remanescentes são mínimas, clinicamente é fácil de ser executada, além do custo e tempo ser menores. Embora existam os benefícios, cuidados devem ser tomados quanto à higienização oral e da prótese, aplicações tópicas de flúor e consultas de retorno ao consultório ${ }^{16}$.

\section{CONCLUSÃO}

Conclui-se que as próteses parciais removíveis overlays são utilizadas para pacientes com desgaste dentário acentuado associado à alteração da dimensão vertical de oclusão. Em contrapartida, estudos ainda precisam ser realizados a fim de assegurar a efetividade e viabilidade desse tipo de prótese, uma vez que, não são aplicáveis em todos os casos clínicos.

\section{CONTRIBUIÇÕES DOS AUTORES}

Matos JDM e Pereira ALC participaram da etapa de conceitos e ideias para a pesquisa em questão, confecção do projeto, definição do tema intelectual, busca bibliográfica, tabulação dos dados, análise dos dados, preparação, revisão e edição do manuscrito. Santos IKS contribuiu na fase de busca bibliográfica nas bases de catalogação bibliográfica. Matos JMM participou na fase de preparação, revisão e edição do manuscrito. Vasconcelos JEL participou da etapa de conceitos e ideias para a pesquisa em questão, confecção do projeto, definição do tema intelectual, preparação, revisão e edição do manuscrito. Figueiredo VMG, participou da etapa de conceitos e ideias para a pesquisa em questão, confecção do projeto, definição do tema intelectual, preparação, revisão e edição do manuscrito.

\section{CONFLITOS DE INTERESSES}

Nenhum conflito financeiro, legal ou político envolvendo terceiros (governo, empresas e fundações privadas, etc.) foi declarado para nenhum aspecto do trabalho submetido (incluindo mas não limitandose a subvenções e financiamentos, conselho consultivo, desenho de estudo, preparação de manuscrito, análise estatística, etc).

\section{REFERÊNCIAS}

1. Batista AUD, Sales JPLA, Farias Neto A, Carreiro AFP. Avaliação do Planejamento de Prótese Parcial Removível em Modelos Recebidos por Laboratórios de João Pessoa, PB. Pesq Bras Odontoped Clin Integr. $2011 ; 11$ (1):53-8. doi: 10.4034/ PBOCl.2011.111.08

2. Goyatá FR, Thomé EMOS, Brum SC, Oliveira RS, Ferreira TFRZ. Tratamento restaurador multidisciplinar - relato de caso clínico. Int J Dent. 2008;7(2):1 42-65.

3. Peterson LJ. Cirurgia oral e maxilofacial contemporânea. $3^{\circ}$ ed. Rio de Janeiro: Guanabara Koogan; 2000.

4. Dekon SFC, Pellizzer EP, Zavanelli AC, Ito L, Resende CA. Reabilitação oral em paciente portador de parafunção severa. Rev Odontol de Araçatuba. 2003;24(1):54-59.

5. Souza JEA, Silva ET, Leles CR. Prótese Parcial Removível Overlay: fundamentos clínicos e relatos de casos. ROBRAC. 2009; 18(47):41-8.

6. Fragoso WS, Troia Junior MG, Valdrighi HC, Chiavini $P$, Oliveira PA. Reabilitação Oral com Prótese Parcial Removível Overlay. RGO. 2005;53(3):243-6.

7. Neto AF, Carreiro AFP, Rizzatti-barbosa CM. A Prótese parcial removível no contexto da odontologia atual. Odontol. Clín.-Cient. 2011 ; 10(2):125-128.

8. Farias $A B L$, Lima LHMA, Costa LJ, Lucena LBS, Farias ABL. Relação entre alteração da dimensão vertical de oclusão e disfunção temporomandibular - avaliação clínica. Braz Dent Sci. 2009;1 2(3): 11 -9. doi: 10.14295/bds.2009.v12i3.633

9. Rodrigues RA. Procedimentos multidisciplinares utilizados na recuperação da DVO durante a reabilitação estética e funcional - relato de caso. Int J Dent. 2010;9(2):96-101.

10.Cura C, Saraçoðlu A, Oztürk B. Prosthetic rehabilitation of extremely worn dentitions: case reports. Quintessence Int. $2002 ; 33(3): 225-30$.

11. Patel MB, Bencharit S. A Treatment Protocol for Restoring Occlusal Vertical Dimension Using an Overlay Removable Partial Denture as an Alternative to Extensive Fixed Restorations: A Clinical Report. Open Dent J. 2009;3(30):2138. doi: $10.2174 / 1874210600903010213$

12. Freitas R, Kaizer OB, Pigozo MN, Cavallari P, Resende DRB. Diagnóstico e Tratamento da Dimensão Vertical de Oclusão Diminuída. RGO. 2006;54(2):161-4.

13. Feltrin PP, Philippi AG, Junior JM, Machado CC, Astolf JA. Dimensões verticais, uma abordagem clínica: revisão de literatura. Rev Odontol Univ Cid Sao Paulo. 2008; 20(3):274-9.

14. Pavarina AC, Machado AL, Vergani CA, Giampaolo ET. Overlay removable partial dentures for a patient with ectodermal dysplasia: A clinical report. J Prosthet Dent. 
$2001 ; 86(6): 574-77$. doi: $10.1067 / \mathrm{mpr} .2001 .119981$

15. Kliemann C, Modaffore PM, Yasuda SH, Nunes IS.

Restabelecimento do Plano Oclusal e da Oclusão dos Dentes

Posteriores com Macroapoios em Prótese Parcial Removível. Rev

Bras Protese Clin Lab. 2000;(6):56-64.

16. Marco RAC, Nogueira JRL, Pavanelli CA, Cardoso FR, Neisser MP. Reabilitação do Plano Oclusal através de Prótese Parcial Removível tipo Overlay. Rev Bras Protese Clin Lab. $2001 ; 3(14): 291-96$. 